\title{
Integrated genomics of susceptibility to alkylator-induced leukemia in mice
}

\author{
Patrick Cahan, Timothy A Graubert
}

\begin{abstract}
Background: Therapy-related acute myeloid leukemia (t-AML) is a secondary, generally incurable, malignancy attributable to chemotherapy exposure. Although there is a genetic component to t-AML susceptibility in mice, the relevant loci and the mechanism(s) by which they contribute to t-AML are largely unknown. An improved understanding of susceptibility factors and the biological processes in which they act may lead to the development of t-AML prevention strategies.

Results: In this work we applied an integrated genomics strategy in inbred strains of mice to find novel factors that might contribute to susceptibility. We found that the pre-exposure transcriptional state of hematopoietic stem/progenitor cells predicts susceptibility status. More than 900 genes were differentially expressed between susceptible and resistant strains and were highly enriched in the apoptotic program, but it remained unclear which genes, if any, contribute directly to t-AML susceptibility. To address this issue, we integrated gene expression data with genetic information, including single nucleotide polymorphisms (SNPs) and DNA copy number variants (CNVs), to identify genetic networks underlying t-AML susceptibility. The 30 t-AML susceptibility networks we found are robust: they were validated in independent, previously published expression data, and different analytical methods converge on them. Further, the networks are enriched in genes involved in cell cycle and DNA repair (pathways not discovered in traditional differential expression analysis), suggesting that these processes contribute to t-AML susceptibility. Within these networks, the putative regulators (e.g., Parp2, Casp9, Polr1b) are the most likely to have a non-redundant role in the pathogenesis of t-AML. While identifying these networks, we found that current CNVR and SNP-based haplotype maps in mice represented distinct sources of genetic variation contributing to expression variation, implying that mapping studies utilizing either source alone will have reduced sensitivity.

Conclusion: The identification and prioritization of genes and networks not previously implicated in t-AML generates novel hypotheses on the biology and treatment of this disease that will be the focus of future research.
\end{abstract}

\section{Background}

Therapy-related acute myeloid leukemia (t-AML) is a secondary malignancy attributable to chemotherapy and/or radiation exposure. $\mathrm{t}$-AML comprises $5-20 \%$ of adult AML cases and its prevalence is increasing along with the size of the population undergoing chemotherapy $[1,2]$. While chemotherapy regimen [3] and genetic background [4] contribute to t-AML, the risk factors are not well understood. Strong evidence for genetic predisposition to t-AML is provided by inherited cancer syndromes such as neurofibromatosis, where germline

\footnotetext{
* Correspondence: graubert@wustl.edu

Department of Internal Medicine, Division of Oncology, Stem Cell Biology Section, Washington University, St. Louis, MO, USA
}

mutations of NF1 are associated with increased risk of t-AML in humans and mice $[5,6]$. Gaining a better understanding of t-AML susceptibility factors is a pressing concern as it may lead to prevention strategies and provide insight into the genesis of de novo AML.

One class of chemotherapeutics associated with t-AML is the alkylators (i.e. melphalan, busulfan, thiotepa). The therapeutic effect of alkylator agents is believed to result from the formation of DNA adducts and single and double-strand breaks, which trigger apoptosis or growth arrest [7]. Based on this presumed mechanism of alkylator action, genes involved in DNA repair [8], response to oxidative stress [9], and drug metabolism [10] have been investigated as mediators of
C Biomed Central

() 2010 Cahan and Graubert; licensee BioMed Central Ltd. This is an Open Access article distributed under the terms of the Creative Commons Attribution License (http://creativecommons.org/licenses/by/2.0), which permits unrestricted use, distribution, and reproduction in any medium, provided the original work is properly cited. 
t-AML susceptibility in candidate gene studies, with largely inconclusive results. A recent study in our lab investigated the genetic basis of $\mathrm{t}$-AML susceptibility using inbred mice [11]. In this study, eight to twelve individual mice from each of 20 inbred strains were treated with the alkylating agent $N$-nitroso- $N$-ethylurea (ENU), a potent mutagen with a propensity to cause AT:TA transversions and AT:GC transitions [12]. Mice were monitored for the development of AML for up to 16 months post ENU exposure. The incidence of AML varied by strain from 0 to $80 \%\left(\mathrm{H}^{2}=0.10\right.$, P-value < 0.001), supporting the hypothesis that there is a strong genetic component in t-AML susceptibility.

We hypothesized that the pre-exposure transcriptional state of hematopoietic stem and progenitor cells, the putative target of leukemogenesis [13], underlies variation in susceptibility to t-AML. A pre-exposure transcriptional basis of susceptibility would be expected if a rapid response is critical in determining a cell's ultimate fate upon mutagen exposure. This hypothesis is consistent with the observation that expression of genes critical to surviving genotoxic stress in yeast does not change after exposure to DNA-damaging agents [14], implying that the necessary factors are already expressed at baseline. A similar situation has been reported in human lymphoblastoid cell lines, in which the pre-exposure transcriptional state of the cell more accurately predicts survival after alkylator treatment than the post-exposure state [15].

In this study, we apply an integrated genomics approach [16] to identify and prioritize genetic and transcriptional networks underlying t-AML susceptibility in mice (Figure 1). By linking expression profiles and complex traits to common genomic loci, this method can ameliorate some of the limitations inherent in genetic association and expression profiling studies [17-21]. When combined with network analysis, this methodology has proven useful in elucidating the biological pathways underlying several complex traits $[22,23]$.

\section{Results}

\section{An integrated genetic map of inbred mouse strains}

Previously, we reported expression quantitative trait loci (eQTLs) in mice using inherited DNA copy number variant regions (CNVRs) as genetic markers [24]. This ciseQTL map did not explicitly include other sources of genetic variation (i.e., SNPs). To derive a more complete map of cis-eQTLs in this population, we used publicly available SNP data from 48 classical inbred strains to map SNP-based eQTLs http://www.broadinstitute.org/ mouse/hapmap/. The SNP resource includes 132,285 SNPs per genome, of which 115,009 we considered informative (as defined in Methods). We used these data to derive haplotype blocks, which are ancestral regions of shared genetic background among strains. Haplotype blocks facilitate trait mapping because they robustly and efficiently represent un-typed genetic variation at a locus. The practical benefit of utilizing haplotype blocks instead of individual SNPs is a reduced number of statistical tests. We used a simple merging algorithm to iteratively join adjacent SNPs into haplotype blocks. This algorithm results in haplotype blocks in which the genotypes of a complete set of SNPs are predictable to a given level of accuracy. We selected a threshold such that for a given block, we can accurately predict the genotype of every SNP in all 48 strains with at most one error (Figure 2A). The 23,884 resulting haplotype blocks are comprised of 1 to 62 SNPs (mean $=4.82$, median $=4)($ Figure $2 \mathrm{~B}$ and Additional File 1). The multi-SNP blocks range in length from 18 to 7,618,246 bp (mean $=83,702$, median $=$ $43,404)$. There are 2 to 6 haplotypes per block (mean $=$ 3.92 , median $=4)($ Figure $2 \mathrm{C})$. Only 21 blocks include haplotypes that are assigned to a single strain. 9,324 blocks have one error, and the remaining 14,560 have zero. Of the 1,262 CNVRs within $250 \mathrm{~Kb}$ of a haplotype block boundary, only one CNVR has a genotype that is tagged $\left(R^{2}>0.80\right)$ by a SNP-based haplotype (Figure $\left.2 \mathrm{D}\right)$. We speculated that the low ability of SNP-derived haplotypes to tag CNVRs was due to the fact that using all 48 classical inbred strains in the haplotype block construction resulted in higher numbers of haplotype labels. Therefore, we also derived a haplotype block map using only the 20 strains from the CNVR study, resulting in a similar inability of SNP-haplotyes to tag CNVRs (data not shown). This suggests that in current databases of genetic variation in the mouse genome, the majority of CNVRs are not captured by SNP resources.

\section{Global pre-exposure transcriptional state of hematopoietic stem and progenitor cells is associated with $\mathrm{t}-\mathrm{AML}$ susceptibility}

We performed gene expression profiling (GEP) in c-kit+/lineage- (KL) bone marrow cells (a population enriched in hematopoietic stem/progenitors) from 20 inbred strains listed as Tier 1-4 from the Mouse Phenome Database [25]. Two-to-three biological replicate arrays were analyzed per strain. This gene expression profiling (GEP) data was previously published [24]. Fifteen of the strains were previously assayed for susceptibility to t-AML after exposure to ENU [11]. Unsupervised clustering of gene expression profiles largely separated susceptible from resistant strains (Figure 3A). The probability that the unsupervised clustering of expression profiles predicts susceptibility status by chance is $<0.01(10,000$ permutations, see Methods and Additional File 2). Further, this clustering is not observed in other tissues that are highly unlikely to be involved in leukemogenesis (i.e., the hypothalamus and adipose tissue), nor does it reflect SNP-based strain 


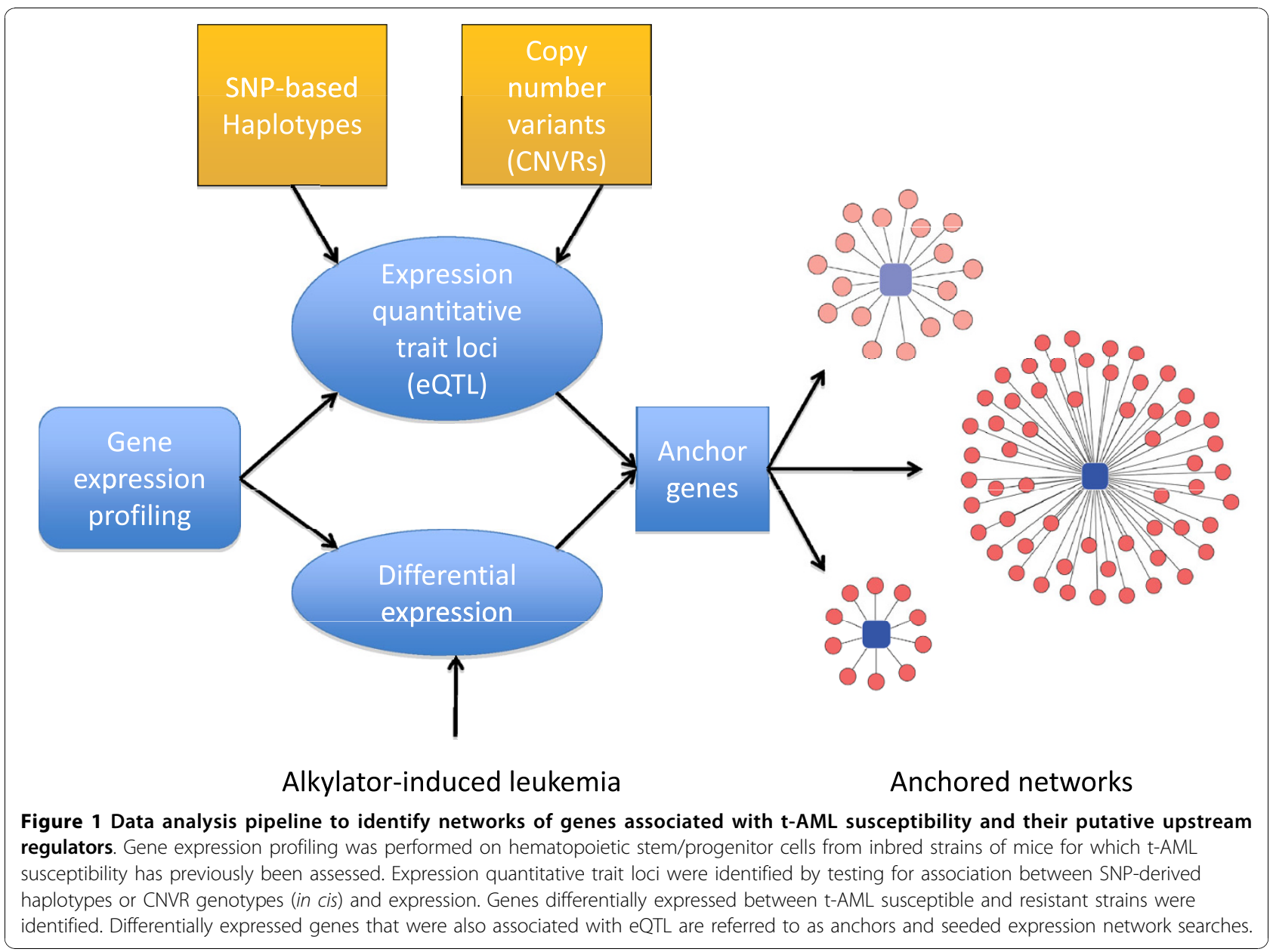

distances (Additional File 2). Taken together, this supports the notion that the KL clustering of susceptible strains is not due to sequence polymorphism affecting target hybridization [26], but rather reflects tissuespecific differences in transcript abundance between inbred strains [27]. Additionally, this observation suggests that the pre-exposure expression differences of many genes, rather than only a few, segregate the KL cells of $\mathrm{t}$-AML susceptible versus resistant strains.

Next, we asked which genes were differentially expressed between susceptible and resistant strains in KL cells. We identified 917 differentially expressed genes (976 probes) at an FDR threshold of 5\% (Additional Files 3 and 4). The differentially expressed genes are enriched in several GO-annotated biological processes (Table 1), including the GO terms 'apoptotic program' and 'nucleotide metabolic process'. The KEGG pathways 'Pyrimidine metabolism' and Colorectal cancer' were also enriched. 'Acute myeloid leukemia' and 'p53 signaling' are biologically plausible pathways that were enriched at least two-fold in the differentially expressed genes, however neither of these pathways passed the FDR $<25 \%$ threshold.
GO-apoptosis-annotated genes included both cell-intrinsic and extrinsic factors (Figure 3B).

\section{Integrated cis-eQTL mapping identifies candidate drivers of t-AML susceptibility}

Our previous eQTL analysis identified 408 expression traits (391 genes) in KL cells that were associated with 214 CNVRs [24]. We repeated this analysis using the 48-strain haplotype resource to map KL expression traits to SNP-based haplotypes. We considered only ciseQTL-associated genes, as it has been shown that transeQTLs contain a large proportion of false positives [28]. We found 127 associations between expression traits and haplotypes, after selecting the most significant association per trait. In the current study, we used the combined set of SNP- and CNVR-based eQTLs to discover and explore genetically driven modules of co-expressed genes associated with $\mathrm{t}$-AML susceptibility.

There are 45 genes ( 45 probes) that are both differentially expressed and linked to at least one eQTL. We refer to these genes as anchors throughout the text. 37 are linked to CNVR-eQTLs; the remaining 8 are linked 


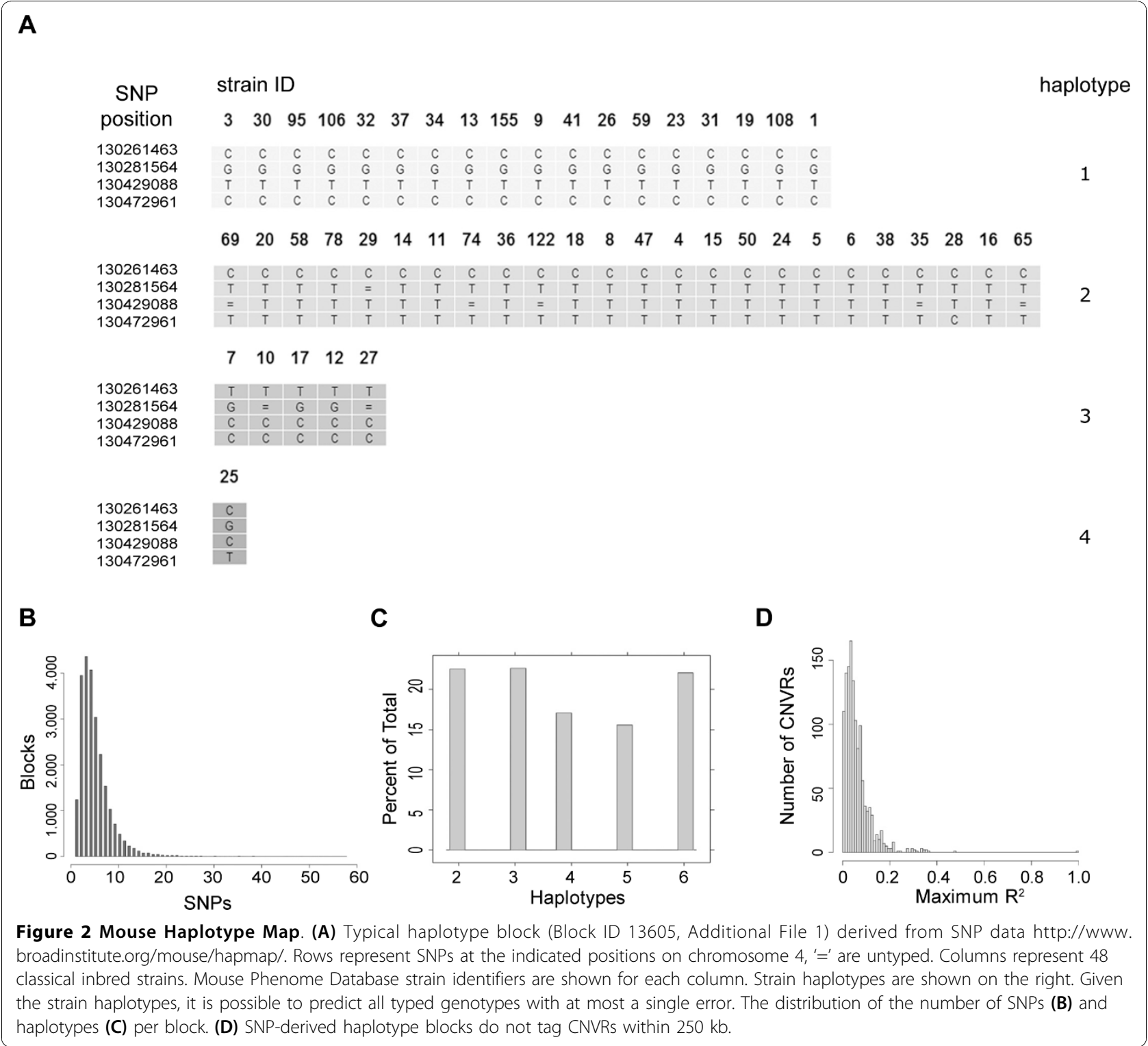

to haplotype-eQTLs. To validate the cis-eQTL associations, we mined publicly available expression data representing hematopoietic stem, progenitor, erythroid and myeloid populations from the BXD recombinant inbred panel [29]. Because this data was generated using the same GEP platform that we used, we were able to ask how our KL population is related to these more purified populations (Additional File 5). As expected, our KL expression profiles cluster most closely with stem and progenitor profiles and are distinct from both erythroid and myeloid lineages. For each anchor gene, we tested the association between BXD genotypes of SNPs within $2 \mathrm{Mb}$ and anchor expression and corrected for multiple testing. We found that 30 of the 45 anchors were significantly associated with at least one SNP within $2 \mathrm{Mb}$ in at least one of the hematopoietic compartments (26 in either Stem or Progenitor), supporting the hypothesis that expression differences of the anchor are caused by locally encoded genetic variation. Of 480 testable eQTLs-transcript associations, 300 (62.5\%) were replicated in at least one of the hematopoietic data sets. KL eQTLs may have failed to validate in the other tissues because they are false positives, because the causative genetic variant does not exist in the BXD strains, or due to tissue-specific expression regulation.

\section{Anchored network analysis identifies t-AML susceptibility expression modules}

Next, we hypothesized that expression differences of anchor genes would cause expression differences 


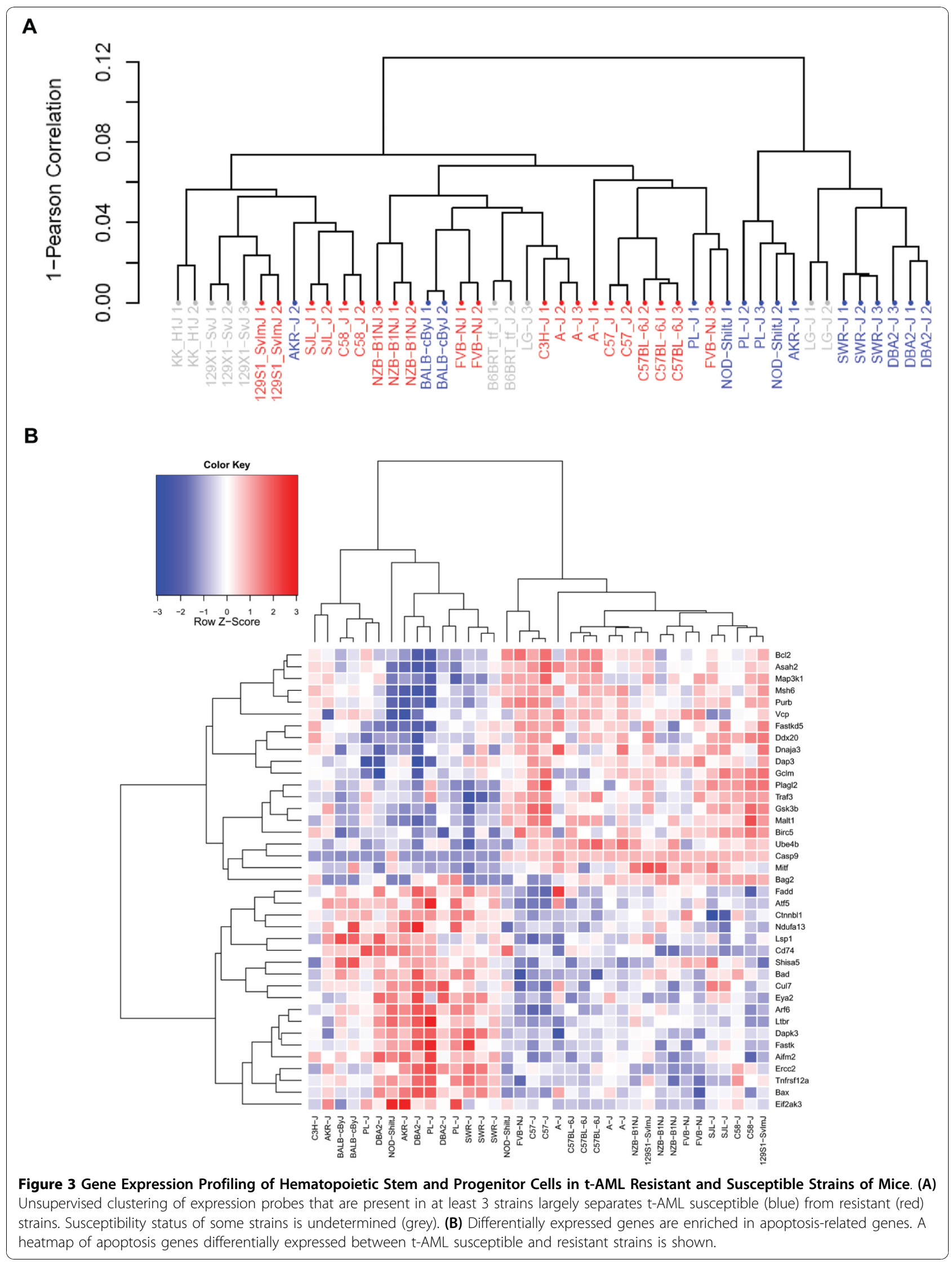


Table 1 Functional enrichment of differentially expressed genes

\begin{tabular}{|c|c|c|c|c|c|}
\hline Annotation & Annotation name & Count & $\begin{array}{r}\text { P-Value } \\
\text { (nominal) }\end{array}$ & $\begin{array}{r}\text { Fold } \\
\text { Enrichment }\end{array}$ & $\begin{array}{r}\text { FDR } \\
(\%)\end{array}$ \\
\hline GO:0008632 & apoptotic program & 11 & $9.58 \mathrm{E}-05$ & 4.71 & 0.17 \\
\hline GO:0006464 & protein modification process & 88 & 1.77E-04 & 1.46 & 0.31 \\
\hline GO:0019318 & hexose metabolic process & 15 & 0.00107053 & 2.76 & 1.88 \\
\hline GO:0005996 & monosaccharide metabolic process & 15 & 0.00129867 & 2.71 & 2.28 \\
\hline GO:0046907 & intracellular transport & 43 & 0.00182315 & 1.63 & 3.18 \\
\hline mmu00240 & Pyrimidine metabolism & 11 & 0.00325706 & 2.98 & 3.99 \\
\hline GO:0031324 & negative regulation of cellular metabolic process & 25 & 0.00230736 & 1.95 & 4.01 \\
\hline GO:0009117 & nucleotide metabolic process & 18 & 0.00246768 & 2.27 & 4.29 \\
\hline GO:0009142 & nucleoside triphosphate biosynthetic process & 9 & 0.00286827 & 3.68 & 4.96 \\
\hline GO:0045934 & $\begin{array}{l}\text { negative regulation of nucleobase, nucleoside, nucleotide and nucleic acid } \\
\text { metabolic process }\end{array}$ & 22 & 0.00335044 & 2.00 & 5.78 \\
\hline GO:0006915 & apoptosis & 39 & 0.00643571 & 1.56 & 10.81 \\
\hline mmu05210 & Colorectal cancer & 10 & 0.00953927 & 2.74 & 11.28 \\
\hline GO:0008637 & apoptotic mitochondrial changes & 5 & 0.00751505 & 6.23 & 12.52 \\
\hline GO:0006396 & RNA processing & 25 & 0.00852521 & 1.76 & 14.08 \\
\hline GO:0009064 & glutamine family amino acid metabolic process & 6 & 0.00927287 & 4.57 & 15.22 \\
\hline GO:0015031 & protein transport & 39 & 0.01063622 & 1.51 & 17.27 \\
\hline GO:0019362 & pyridine nucleotide metabolic process & 5 & 0.01370052 & 5.27 & 21.69 \\
\hline GO:0008219 & cell death & 39 & 0.01444197 & 1.48 & 22.73 \\
\hline GO:0016481 & negative regulation of transcription & 19 & 0.01461908 & 1.85 & 22.98 \\
\hline
\end{tabular}

Count: Number of genes differentially expressed between t-AML-resistant and -susceptible inbred strains of mice with given annotation.

(in trans) on multiple downstream genes (targets). For each anchor, we identified correlated expression profiles (FDR $<1 \%$ ), resulting in 30 sets of co-expressed genes or modules. The number of targets per module ranged from 3 to $607($ mean $=113$, median $=72)$. We reasoned that true response genes will exhibit association with anchor expression even when the remaining genome is randomly shuffled, as it is in the BXD recombinant inbred cross. For each module, we tested the association between expression of the anchor and each response transcript in each of the BXD hematopoietic populations. We removed target genes from modules that were not associated with anchor gene expression in at least one population (FDR < 25\%) (Table 2). We also used a Weighted Gene Co-expression Network Analysis (WGCNA) [30,31], to derive modules of correlated genes independent of linkage to eQTLs. We filtered these modules on the basis of their reproducibility in the BXD dataset and compared the resulting modules with the anchored expression networks. The WGCNA modules are highly similar to the anchored modules in gene content, suggesting that the discovered co-expression structure is robust to different algorithms (data not shown).

The expression of each anchor gene is, by definition, associated with susceptibility status. However, the strength of the association between the target genes of an anchored module and susceptibility is unknown. To determine these values, we first computed eigengenes from each module [31]. Then, we ranked anchored modules according to differential expression of the module's eigengene and susceptibility status. Using both KEGG and GO annotations, we found that 8 anchored modules were enriched in at least one annotation. We visualized the anchored susceptibility modules as both heatmaps of eigengene values (Figure 4A) and networks (Figure 4B), displaying the correlation between anchored modules and the strength of association between anchored modules and susceptibility status. We also visualized one of the anchored susceptibility networks, focusing on a biologically compelling module (Figure 4C).

\section{Discussion}

There is accumulating evidence that many genetic contributors to complex traits are not protein-coding changes [32,33]. If true, then other classes of genetic events that can affect phenotype must, at some level, impact gene expression (i.e., eQTLs). Hypothesizing that such events contribute to t-AML susceptibility, we took an integrated genomics approach to identify and prioritize candidate transcriptional networks. The first step in this approach was to identify eQTLs in hematopoietic stem and progenitor cells, the likely target of leukemic transformation. Previously, we described a CNVR eQTL map in classical inbred mice [24]. In the current work, we expanded this map to include SNP-based haplotype eQTLs. In deriving the mouse haplotype map, we found 
Table 2 Anchored susceptibility modules

\begin{tabular}{|c|c|c|c|c|c|c|c|c|c|c|}
\hline \multirow[b]{2}{*}{ Module } & \multirow[b]{2}{*}{ Anchor Gene } & \multirow[b]{2}{*}{$\mathrm{KL}$} & \multicolumn{4}{|c|}{ BXD } & \multirow[b]{2}{*}{$\mathbf{N}^{*}$} & \multirow[b]{2}{*}{ P-value } & \multirow[b]{2}{*}{ Top GO } & \multirow[b]{2}{*}{ Top KEGG } \\
\hline & & & KLS & KLS- & $\mathrm{Gr}$ & Ter & & & & \\
\hline$\overline{A \_1}$ & LOC634046 & 460 & 43 & 2 & 23 & 199 & 236 & 2.25 & GO:0032940 & $\mathrm{NE}$ \\
\hline A_2 & scl41743.2_361 & 402 & 140 & 272 & 207 & 19 & 329 & 1.09 & GO:0019219 & map04120 \\
\hline A_3 & Gl_38089999 & 38 & 4 & 1 & 0 & 2 & 7 & 1.72 & $\mathrm{NE}$ & $\mathrm{NE}$ \\
\hline A_4 & A330106M24Rik & 4 & 3 & 3 & 3 & 1 & 4 & 1.82 & $\mathrm{NE}$ & $\mathrm{NE}$ \\
\hline A_6 & Ociad2 & 132 & 63 & 3 & 16 & 10 & 75 & 1.5 & GO:0043666 & $\mathrm{NE}$ \\
\hline A_7 & Gl_46852192-I & 97 & 1 & 15 & 1 & 50 & 54 & 1.95 & GO:0051329 & $\mathrm{NE}$ \\
\hline A_9 & Zfp862 & 106 & 13 & 19 & 1 & 0 & 26 & 2.3 & $\mathrm{NE}$ & $\mathrm{NE}$ \\
\hline A_12 & A630001G21Rik & 112 & 79 & 50 & 38 & 48 & 104 & 2.38 & GO:0045449 & $\mathrm{NE}$ \\
\hline A_14 & Aste1 & 102 & 7 & 48 & 0 & 1 & 52 & 2.67 & $\mathrm{NE}$ & $\mathrm{NE}$ \\
\hline A_16 & Ckap2l & 607 & 4 & 116 & 289 & 4 & 356 & 1.28 & GO:0006281 & map04070 \\
\hline A_17 & H2-Ke6 & 238 & 12 & 109 & 2 & 81 & 152 & 1.93 & $\mathrm{NE}$ & $\mathrm{NE}$ \\
\hline A_20 & Dusp16 & 91 & 45 & 4 & 43 & 30 & 72 & 2.08 & $\mathrm{NE}$ & $\mathrm{NE}$ \\
\hline A_21 & scl0217069.13_16 & 58 & 3 & 4 & 21 & 4 & 29 & 2.72 & $\mathrm{NE}$ & $\mathrm{NE}$ \\
\hline A_22 & Atf7ip & 39 & 1 & 1 & 9 & 10 & 19 & 2.61 & $\mathrm{NE}$ & $\mathrm{NE}$ \\
\hline A_23 & Snrpn & 4 & 2 & 2 & 0 & 1 & 3 & 0.94 & $\mathrm{NE}$ & $\mathrm{NE}$ \\
\hline A_24 & Atp6v0e2 & 78 & 4 & 5 & 1 & 0 & 5 & 2.91 & $\mathrm{NE}$ & $\mathrm{NE}$ \\
\hline A_25 & Gimap7 & 30 & 3 & 2 & 0 & 6 & 9 & 2.35 & $\mathrm{NE}$ & $\mathrm{NE}$ \\
\hline A_26 & Pdzk1ip1 & 79 & 39 & 31 & 0 & 20 & 57 & 2.35 & $\mathrm{NE}$ & $\mathrm{NE}$ \\
\hline A_27 & Polr1b & 27 & 3 & 10 & 4 & 2 & 12 & 3.11 & $\mathrm{NE}$ & $\mathrm{NE}$ \\
\hline A_28 & Magohb & 65 & 55 & 35 & 24 & 47 & 61 & 0.97 & GO:0044242 & $\mathrm{NE}$ \\
\hline A_30 & Sox13 & 34 & 18 & 25 & 3 & 1 & 29 & 1.45 & GO:0006631 & $\mathrm{NE}$ \\
\hline A_32 & Ptcd3 & 18 & 7 & 14 & 5 & 4 & 17 & 2.68 & $\mathrm{NE}$ & $\mathrm{NE}$ \\
\hline A_33 & Casp9 & 37 & 1 & 0 & 6 & 1 & 6 & 3.22 & $\mathrm{NE}$ & $\mathrm{NE}$ \\
\hline A_34 & Ctsf & 223 & 53 & 123 & 8 & 71 & 169 & 2.34 & $\mathrm{NE}$ & $\mathrm{NE}$ \\
\hline A_36 & scl46617.10.1_4 & 13 & 4 & 3 & 8 & 5 & 10 & 2.5 & $\mathrm{NE}$ & $\mathrm{NE}$ \\
\hline A_37 & Parp2 & 88 & 21 & 21 & 17 & 19 & 42 & 2.05 & $\mathrm{NE}$ & $\mathrm{NE}$ \\
\hline A_38 & Hdhd3 & 178 & 72 & 2 & 70 & 1 & 102 & 1.78 & $\mathrm{NE}$ & $\mathrm{NE}$ \\
\hline A_39 & 5830417I10Rik & 5 & 1 & 3 & 1 & 1 & 3 & 2.07 & $\mathrm{NE}$ & NE \\
\hline A_41 & Prcp & 3 & 2 & 1 & 2 & 2 & 3 & 2.11 & $\mathrm{NE}$ & $\mathrm{NE}$ \\
\hline A_43 & Ggcx & 7 & 6 & 7 & 5 & 3 & 7 & 2.53 & $\mathrm{NE}$ & $\mathrm{NE}$ \\
\hline
\end{tabular}

$\mathrm{KL}$ : Number of probes significantly associated with anchored gene expression in kit+/lineage- (KL) cells.

BXD: Number of probes in module significantly associated with anchored gene expression in BXD KLS (Sca+/kit+/lineage-, HSC), KLS- (Sca-/kit+/lineage-, Progenitor), Gr-1+ (Myeloid), or Ter-119+ (Erythroid) cells.

*, Number of probes in module significantly associated with anchored gene expression in at least one BXD data set.

\#, -Log10(P-value) for association with t-AML susceptibility.

Top GO/KEGG, annotations significantly enriched in each module; $\mathrm{NE}$, not enriched.

surprisingly little correlation between haplotypes and neighboring CNVRs. This is in contrast to human studies, where nearly $75 \%$ of common CNVRs are estimated to be in linkage disequilibrium with neighboring SNPs [34]. This suggests that at the currently available resolution and coverage (and genotyping accuracy), mouse haplotypes and CNVRs represent distinct sources of genetic information. We found two-fold more CNVR eQTLs than haplotype-based eQTLs (401 vs. 167). It is tempting to speculate that this difference in eQTL types is because CNVRs have a stronger impact on expression in cis and therefore are more likely to be detected as eQTLs. However, the difference could largely be due to the reduced power to detect haplotype eQTLs because of the exacerbated multiple testing problem that comes with performing approximately 20 times more statistical tests. In total, greater than $60 \%$ of the eQTLs were reproducible in an independent dataset.

The second step in the integrated approach was to find genes differentially expressed between t-AML susceptible and resistant strains. Because unsupervised clustering using all expressed transcripts grouped strains by susceptibility status, we expected to find a large number of genes associated with susceptibility. Greater than 7\% of the expressed transcripts are differentially expressed $(976 / 13,496)$. These genes are enriched in several, independent biological processes, most notably apoptosis. Among the differentially expressed intrinsic apoptosis 


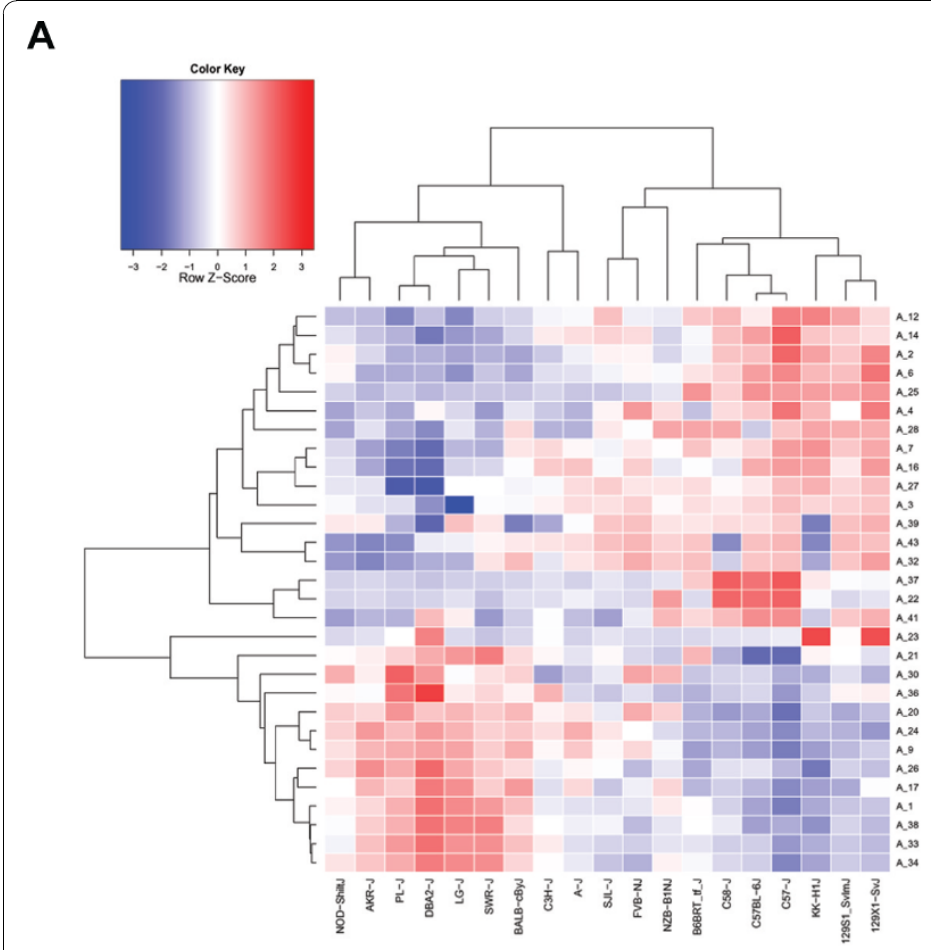

B

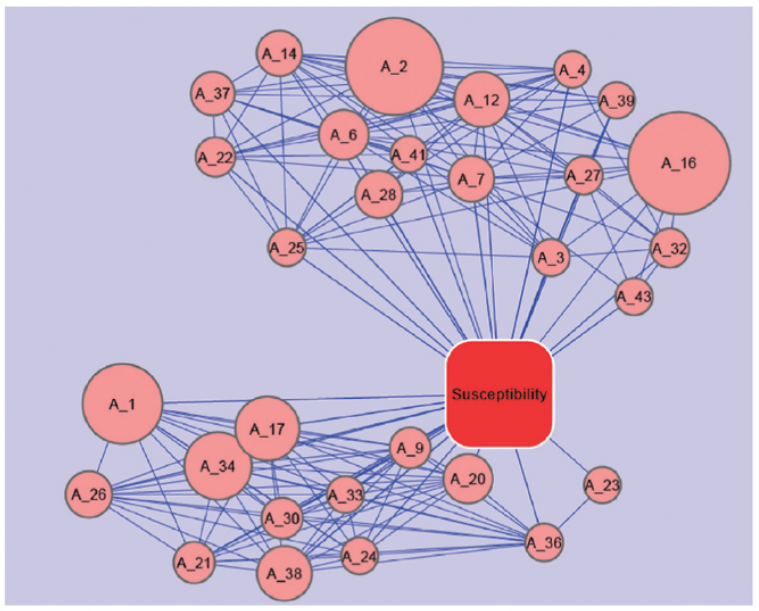

C

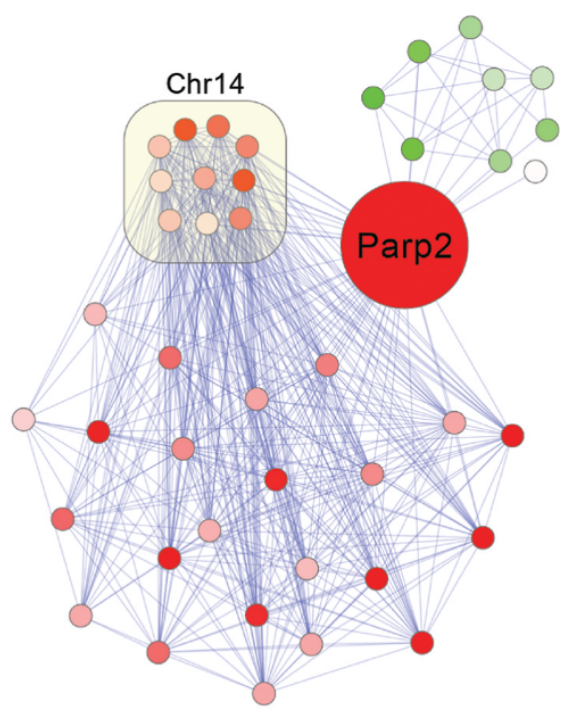

Figure 4 Anchored Susceptibility Networks. (A) Heatmap of anchored module eigengenes. For clarity, eigengene values were averaged by strain, and each module was row-normalized. Module Eigengenes are either positively (bottom half) or negatively (top half) correlated with susceptibility status. (B) Network view of anchored modules. Anchored modules are represented as nodes. Edges between modules represent network eigenegene correlation. Low and negative correlations are not shown for clarity. Edges between the 'Susceptibility' and anchored network nodes represent association between network eigengenes and susceptibility status. Node size indicates the number of response genes in the anchored network. The top super-module corresponds to the top half of the module heatmap displayed in panel A. Likewise, the bottom super-module corresponds to the bottom half of the module heatmap. (C) Module A_37, includes 10 genes on chromosome 14 located within $7 \mathrm{Mb}$ of a CNVR. Green nodes represent genes with lower expression in susceptible strains, red nodes represent genes with higher expression in susceptible strains. Correlations among response genes, represented as edges, are only displayed for those relationships where the Pearson correlation $>0.5$. 
genes are Caspase 9 (Casp9), B-cell leukemia/lymphoma $2(\mathrm{Bcl}-2), \mathrm{BCL} 2$-associated agonist of cell death $(\mathrm{Bad})$, BCL2-associated X protein (Bax), and mutS homolog 6 (Msh6). Msh6 is a member of the mutSa DNA mismatch recognition complex that has been shown to mediate apoptosis in certain contexts [35,36]. Notably, the absence of mutS $\alpha$ activity in myeloid progenitors results in the complete loss of O6-methylguanine (O6MeG)-mediated cytotoxicity [37]. That resistant strains have higher expression of Msh6 suggests that upon alkylator exposure, resistant strains may recognize DNA damage and respond appropriately (i.e. undergo apoptosis) whereas the KL cells of susceptible strains may tend to survive, accumulate mutations, and become transformed. In KL cells, almost all susceptible strains have no detectable expression of Casp9, an initiator of programmed cell death, suggesting that these cells (lowto-no Casp9 expression) are less primed for Casp9dependent apoptosis. Although knockout of Casp9 in mice is embryonic lethal, presumably due to severe brain development defects [38,39], Casp 9 is not required for apoptosis during normal hematopoiesis [40]. Therefore, inbred strains may exhibit genetically-driven yet tissue-specific differences in apoptosis such that hematopoietic cells of susceptible strains are relatively protected from cell death after exposure to genotoxic stress.

Differential expression and gene enrichment analysis highlighted several biologically plausible pathways that may underlie t-AML susceptibility. However, it remained unclear which pathway members, if any, are causal contributors to the phenotype, as illustrated by the complex expression patterns of the intrinsic apoptosis genes. More broadly, the role and relative importance of each of the 917 differentially expressed genes in susceptibility remained undetermined. We hypothesized that among the 917 differentially expressed genes would be a subset in which expression variation is caused by cis-encoded genetic variation. Further, we posited that these 'anchor' genes cause expression variation of multiple downstream genes, which collectively are associated with t-AML susceptibility (or resistance). The mechanisms by which anchors might act in trans are varied. They include altered transcription factor abundance and homeostatic or compensatory forces within and between biological pathways. Regardless of the mechanisms of action, the identification of putative events that influence susceptibility and their linkage to gene networks forms a powerful and practical strategy to both find biological pathways underlying cancer susceptibility and to prioritize candidate mediators. Therefore, as the third step in the integrated genomics approach, we identified networks of genes that are significantly correlated with candidate susceptibility anchors. To validate the networks, we used independent gene expression data from multiple hematopoietic populations, trimming the networks of response genes whose expression was not reproduced.

One of the benefits of the integrated genomics approach is that it can implicate biological processes that would not have been detected using differential expression alone. The susceptibility networks that we identified are enriched in genes involved in DNA repair, base excision repair, apoptosis, and cell cycle, among other annotations. A second potential benefit of the integrated approach is that it differentiates between upstream (anchors) and response genes, an advantage over existing approaches that derive gene regulatory networks from expression data alone. While this is a hypothesis that remains to be tested, the identification of candidate upstream factors will be useful in prioritizing among apoptosis-related genes for experimental validation. For example, although Casp 9 and Bcl2 are differentially expressed, Casp 9 is also the candidate anchor of module A_33, the module most strongly associated with susceptibility status. We speculate that perturbation of candidate anchors, such as Casp9, are more likely to be informative in elucidating susceptibility than response genes (i.e. $B c l 2$ ).

Network analysis allowed us to predict the function of uncharacterized genes. For example, A630001G21Rik is expressed primarily in primitive hematopoietic and B-cells [41], yet its function is undetermined. Our analysis places it as the anchor of module A_12, which is enriched in apoptosis-related genes, including Bcl2. Therefore, A630001G21Rik may play previously unknown role in regulation of $B c l 2$ expression and apoptosis activity. Similarly, Cytoskeleton-associated protein-like 2 (Ckap2l) is the anchor of the largest module, A_16, enriched in both cell cycle and DNA repair genes. Although $\mathrm{Ckap} 2 l$ is highly expressed in hematopoietic progenitors [41], its functions are unknown. Its closest ortholog, Ckap2, is highly expressed in mouse stem cell lines and has detectable expression in hematopoietic progenitors, bone marrow, osteoclasts, osteoblasts, and macrophages [41]. There is a growing body of literature suggesting that Ckap2 (also known as Tumor-associated microtubule-associated protein) is involved in cell cycle progression [42-44]. It is possible that Ckap2l contributes to cell cycle regulation in HSCs and progenitors, and that genetic disturbances of its expression impact t-AML susceptibility. Experiments that perturb expression of anchor genes such as Casp 9 and Ckap $2 l$ to assess their impact on module expression and activity are the next logical steps in determining the role of candidate networks in susceptibility. If such experiments demonstrate a causal link between anchor genes and module expression, then moving forward to formally define their role in leukemia will be warranted. 
A drawback to the anchored network approach, as currently implemented, is that it assumes there is only a single anchor per module. In cases where CNVRs disrupt regulatory elements, it is possible that a single genetic event impacts the expression of multiple neighboring genes. For example, in module A_37 (Figure 4C) we found 10 response genes within $7 \mathrm{Mb}$ of a CNVR. This module warrants special attention because it includes poly (ADP-ribose) polymerase family member 2 (Parp2, the anchor) and apurinic/apyrimidinic endonuclease 1 (Apex1), both members of the base excision repair pathway $[45,46]$. Both genes have lower expression in susceptible strains, again suggesting that lowered overall DNA damage response promotes susceptibility.

A caveat to the current work is that maps of genetic variation in the mouse genome are incomplete, a knowledge gap that promises to be filled by more informative SNP arrays [47] and next-generation sequencing [48,49]. It is possible that un-captured genetic variants may be the ultimate cause of the observed co-expression networks. These variants may mediate their impact through mechanisms other than altering the expression of anchors. In the extreme case, all modules may not be controlled by anchor expression, but by undetected causes. Nevertheless, the modules themselves are still informative in that they describe sets of coordinately regulated genes that, collectively, are associated with both t-AML susceptibility and biologically plausible processes and pathways.

\section{Conclusions}

To our knowledge, this is the first report of an integrated genomics approach to dissect the role of the preexposure transcriptional state in t-AML susceptibility. From a clinical perspective, t-AML is important because the response to treatment is poor and survival is short [3]. But because t-AML is a clinically-induced malignancy, it is by definition preventable. Therefore, a longterm goal in this field is to gain sufficient understanding of susceptibility factors in order to make worthwhile the personalization of chemotherapeutic regimens based on t-AML risk. The transcriptional networks and their candidate anchors described here are an important early step towards gaining such an understanding.

\section{Methods}

\section{Construction of SNP-based haplotype map}

Genomic coordinates of 1,333 CNVRs were mapped from $\mathrm{mm} 8$ to $\mathrm{mm} 9$ using liftOver. 31 CNVRs were unmapped and dropped from further analysis. To derive haplotype blocks, SNPs for the haplotype map construction were downloaded from the Broad Institute http:// www.broadinstitute.org/mouse/hapmap/. Only SNPs from 48 non-wild-derived strains were used. SNPs that were contained within CNVRs, had minor allele frequencies $<5 \%$, or were not genotyped in $25 \%$ or more of strains were considered uninformative and were excluded from further analysis. The following steps were performed to simultaneously group SNPs into blocks and to assign haplotype to strains:

(1) Begin with the first informative SNP on a chromosome.

(2) If the number of SNPs in the current block is 1 then go to (3). Otherwise, go to (4).

(3) Group strains by genotype and add the next consecutive SNP to the current block.

(4) Cluster strains by SNP-based distance using Partitioning Around Medioids[50] (number of clusters = 2 to 6$)$.

(5) Assign haplotype labels to strains based on the clustering with the maximum average silhouette [50].

(6) Derive consensus haplotypes. For each haplotype cluster, a consensus haplotype is defined as the string comprised of the most frequent genotype at each SNP position.

(7) Compare the consensus haplotypes to the actual SNP genotypes.

(8) If the number of errors is greater than 1 then go to (9), otherwise go to (10).

(9) Remove the most recently added SNP from the current block. Store the haplotyping results from the previous iteration. Start a new block with the current SNP. Go to (3).

(10) Add the next consecutive SNP to the current block. Go to (4). If there are no more SNPs on the current chromosome, select a new chromosome and go to (2). The computation is complete when all chromosomes have been analyzed.

SNP-based distances between strains are computed as the sum of SNP differences between strains. The range of number of allowable haplotypes per block was selected based on the estimated number of ancestral haplotypes [51]. Pooled multi-allelic $\mathrm{R}^{2}$ was computed based on haplotype frequencies [52].

\section{Integrated expression QTL mapping \\ Pre-processing}

GEP expression profiling was previously described [24] and is available at GEO under accession GSE10656. This data is referred to as kit+/lineage- (KL) throughout the text. Hypothalamus and adipose tissue expression data were obtained from GEO (accessions GSE5961 and GSE8028, respectively). For clustering and network analysis, probes were first filtered based on detection. In the KL data, a probe was considered detected in a sample if its signal was greater than a set of negative 
controls on the Illumina array. 13,496 probes were detected in all biological replicates of at least three strains (excluding $\mathrm{C} 3 \mathrm{H}$, for which only one array was analyzed). Only the 14,871 and 10,040 probes that were detected as present in at least $25 \%$ of the strains in the hypothalamus and adipose data sets, respectively, were kept for clustering analysis.

\section{Cluster analysis}

Unsupervised hierarchical clustering was performed with R's hclust function, using 1-Pearson correlation as the distance metric and the complete linkage method for node merging. To assess the non-randomness of the strains clustering according to susceptibility status, we computed the ratio of the mean of the distances among susceptible strains to the mean of the distances between all susceptible and resistant strains. Then, we permuted the strain labels 10,000 times, and recomputed the ratio of distances. The P-value of the observed clustering is the number of random permutations in which the distance test statistic $>=$ observed distance test statistic divided by 10,000 . This analysis was performed on the median expression profiles of strain replicates, only in those strains in which the susceptibility status is known. SNP clustering was based on strain-strain pair-wise distances computed by counting the number of SNPs that differ between each of the strains divided by the total number of SNPs that are typed in both strains.

\section{Differential expression analysis}

Strains with unknown susceptibility status were not included in the differential expression analysis. We used the limma package in $\mathrm{R}$ to model the expression of each gene with coefficients representing strain replicates and susceptibility status $[53,54]$ and the false discovery rate (FDR) was estimated using q-value [55]. All of the 976 significant probes were detected as present in at least $50 \%$ of either the susceptible or resistant strains. When a gene is targeted by more than one probe, only the most significant differentially expressed probe was used for visualization. Association of module eigengenes with susceptibility was tested in the same way as differential expression. Enrichment analysis was performed using DAVID [56]. Only the GO annotations Biological Process 5 and KEGG pathways were assessed. We only report annotations that pass an FDR threshold $<25 \%$. Expression data from all 20 strains previously profiled were used in expression network analysis. Anchored expression networks were identified by searching for probes that exhibited expression profiles that were significantly correlated with anchor gene expression at an FDR threshold $<1 \%$.

\section{Expression quantitative trait locus mapping}

CNVR eQTLs previously identified were used in this analysis [24] and eQTLs based on SNP haplotypes were identified using the haplotype association method with weighted strain permutation to account for strain relatedness [57-59]

\section{Analysis of coexpression networks}

Normalized gene expression data used for validation of eQTLs and anchored modules was downloaded from GEO (GSE18067). This data set includes profiling on sorted (purified) hematopoietic stem, progenitor, myeloid and erythroid populations from female BXD recombinant inbred mice [29]. Only detection calls, coded as 0 for absent or 1 for present, were used to globally compare our KL data to the BXD data. Clustering was performed using the same parameters as described above for the KL data. KL eQTLs were validated by testing the association between the genotypes of SNPs within $2 \mathrm{Mb}$ of anchor genes and driver gene expression in each compartment separately. Genotypes were treated as factors in a linear model of driver gene expression. $\mathrm{P}$-values of the resulting F-statistics were adjusted for multiple testing using Holm's method [60]. Drivers that had corrected P-values $<0.05$ in at least one compartment were considered validated. Assessing the reproducibility of the association between driver and response gene expression was performed in a similar manner. A linear model of response gene expression was fit with driver gene expression as the dependent variable (one model per driver-response gene pair per compartment). In this case, Benjamini and Hochberg's method to control the false discovery rate was applied to the resulting p-values [61]. WGCNA analysis was performed as previously described using the $\mathrm{R}$ package WGCNA [31]. Briefly, $\beta$ values for calculating the weighted network adjacency were selected based on the power at which the scale law $R^{2}$ exceeded 0.9. Weighted adjacency matrices were computed, modules were defined using the cut Tree Dynamic function (which selects good dendrogram cutoffs) and similar modules were merged using merge Close Modules (which compensates for the high sensitivity of WGCNA). Eigengenes were computed as the first principal component of a module's expression matrix. Eigengenes were tested for differential expression between susceptible and resistant strains, as described above for individual genes.

\section{Additional material}

Additional file 1: Supplementary Table S1. SNP-derived haplotype blocks in 48 inbred mouse strains.

Additional file 2: Dendrograms showing clustering of strains by gene expression profile or SNP-derived haplotype blocks. (A) Unsupervised clustering of strains using the strain median expression profile in $\mathrm{KL}$ cells groups strains by t-AML susceptibility status to an extent greater than expected by chance (see text), and differently than when clustering gene expression profiles of the hypothalamus (B),

adipose tissue (C), or when clustering based on SNP-based distance (D). 
Additional file 3: Heatmap of genes differentially expressed in KL cells from t-AML susceptible vs. resistant strains of mice. 917 genes (976 probes) are differentially expressed between t-AML susceptible (SWR/J, DBA2/J, PL/J, AKR/J, BALB/CByJ) and t-AML resistant (C58/J, C57/J, FVB/J, C57BL/6J, A/J, NZB/J, C3H/HeJ, SJL/J, and 129S1/SvImJ) mice.

Additional file 4: Supplementary Table S2. Annotation of genes differentially expressed between t-AML susceptible and resistant strains of mice.

Additional file 5: Unsupervised clustering of expression profiles of purified hematopoietic compartments. BXD populations are indicated by the enriched population: erythrocytes (Ter119+, orange), myeloid lineage (Gr1+,green), hematopoietic stem cells (Lineage-Kit+Sca1+, red), and progenitors (Lineage-Kit+Sca1-, blue). Hematopoietic stem and progenitors from classical inbred strains are indicated by inbred strain name (Lineage-cKit+, navy blue). Each population forms a distinct cluster, with $\mathrm{KL}$ cells grouping most closely with stem and progenitor cells.

\section{List of Abbreviations}

(t-AML): therapy-related acute myeloid leukemia; (CNV): DNA copy number variation; (eQTL): expression quantitative trait loci; (SNPs): single nucleotide polymorphisms; (ENU): N-nitroso-N-ethylurea; (CNVRs): copy number variant regions; (GEP): gene expression profiling; (KL): c-kit+/lineage-; (FDR): false discovery rate; (WGCNA): Weighted Gene Co-expression Network Analysis; (Casp9): Caspase 9; (BCl-2): B-cell leukemia/lymphoma 2; (Bad): BCL2associated agonist of cell death; (Bax): BCL2-associated X protein; (Msh6): mutS homolog 6; (O6MeG): O6-methylguanine; (Ckap2): Cytoskeletonassociated protein-like 2; (Parp2): poly (ADP-ribose) polymerase family member 2; (Apex1): apurinic/apyrimidinic endonuclease 1.

\section{Acknowledgements}

P.C. was supported in part by the National Human Genome Research Institute (T32 HG000045) and a Kauffman Fellowship. This work was supported by the NIH (P01CA101937). We thank Nancy L. Saccone for helpful discussions.

\section{Authors' contributions}

PC and TG designed the study, performed analysis, and wrote the manuscript. Both authors read and approved the final draft.

Received: 29 April 2010 Accepted: 17 November 2010 Published: 17 November 2010

\section{References}

1. Leone G, Voso MT, Sica S, Morosetti R, Pagano L: Therapy related leukemias: susceptibility, prevention and treatment. Leuk Lymphoma 2001, 41(3-4):255-276.

2. Leone G, Pagano L, Ben-Yehuda D, Voso MT: Therapy-related leukemia and myelodysplasia: susceptibility and incidence. Haematologica 2007 92(10):1389-1398.

3. Larson RA, Le Beau MM: Therapy-related myeloid leukaemia: a model for leukemogenesis in humans. Chem Biol Interact 2005, 153-154:187-195.

4. Knoche E, Mc Leod HL, Graubert TA: Pharmacogenetics of alkylatorassociated acute myeloid leukemia. Pharmacogenomics 2006, 7(5):719-729.

5. Side L, Taylor B, Cayouette M, Conner E, Thompson P, Luce M, Shannon K Homozygous inactivation of the NF1 gene in bone marrow cells from children with neurofibromatosis type 1 and malignant myeloid disorders. N Engl J Med 1997, 336(24):1713-1720.

6. Jacks T, Shih TS, Schmitt EM, Bronson RT, Bernards A, Weinberg RA: Tumour predisposition in mice heterozygous for a targeted mutation in Nf1. Nat Genet 1994, 7(3):353-361.

7. Meikrantz W, Bergom MA, Memisoglu A, Samson L: O6-alkylguanine DNA lesions trigger apoptosis. Carcinogenesis 1998, 19(2):369-372.

8. Seedhouse C, Bainton R, Lewis M, Harding A, Russell N, Das-Gupta E: The genotype distribution of the XRCC1 gene indicates a role for base excision repair in the development of therapy-related acute myeloblastic leukemia. Blood 2002, 100(10):3761-3766.
9. Allan JM, Wild CP, Rollinson S, Willett EV, Moorman AV, Dovey GJ, Roddam PL, Roman E, Cartwright RA, Morgan GJ: Polymorphism in glutathione S-transferase P1 is associated with susceptibility to chemotherapy-induced leukemia. Proc Natl Acad Sci USA 2001, 98(20):11592-11597.

10. Larson RA, Wang Y, Banerjee M, Wiemels J, Hartford C, Le Beau MM, Smith MT: Prevalence of the inactivating $609 \mathrm{C}->\mathrm{T}$ polymorphism in the $\mathrm{NAD}(\mathrm{P}) \mathrm{H}$ :quinone oxidoreductase (NQO1) gene in patients with primary and therapy-related myeloid leukemia. Blood 1999, 94(2):803-807.

11. Fenske TS, MC Mahon C, Edwin D, Jarvis JC, Cheverud JM, Minn M, Mathews V, Bogue MA, Province MA, McLeod HL, et al: Identification of candidate alkylator-induced cancer susceptibility genes by whole genome scanning in mice. Cancer Res 2006, 66(10):5029-5038.

12. Noveroske JK, Weber JS, Justice MJ: The mutagenic action of N-ethyl-Nnitrosourea in the mouse. Mamm Genome 2000, 11(7):478-483.

13. Thirman MJ, Larson RA: Therapy-related myeloid leukemia. Hematol Oncol Clin North Am 1996, 10(2):293-320.

14. Birrell GW, Brown JA, Wu HI, Giaever G, Chu AM, Davis RW, Brown JM: Transcriptional response of Saccharomyces cerevisiae to DNA-damaging agents does not identify the genes that protect against these agents. Proc Natl Acad Sci USA 2002, 99(13):8778-8783.

15. Fry RC, Svensson JP, Valiathan C, Wang E, Hogan BJ, Bhattacharya S, Bugni JM, Whittaker CA, Samson LD: Genomic predictors of interindividual differences in response to DNA damaging agents. Genes Dev 2008, 22(19):2621-2626.

16. Schadt EE: Molecular networks as sensors and drivers of common human diseases. Nature 2009, 461(7261):218-223.

17. Meng H, Vera I, Che N, Wang X, Wang SS, Ingram-Drake L, Schadt EE, Drake TA, Lusis AJ: Identification of Abcc6 as the major causal gene for dystrophic cardiac calcification in mice through integrative genomics. Proc Natl Acad Sci USA 2007, 104(11):4530-4535.

18. Wang SS, Shi W, Wang X, Velky L, Greenlee S, Wang MT, Drake TA, Lusis AJ: Mapping, genetic isolation, and characterization of genetic loci that determine resistance to atherosclerosis in $\mathrm{C} 3 \mathrm{H}$ mice. Arterioscler Thromb Vasc Biol 2007, 27(12):2671-2676.

19. Schadt EE, Monks SA, Drake TA, Lusis AJ, Che N, Colinayo V, Ruff TG, Milligan SB, Lamb JR, Cavet G, et al: Genetics of gene expression surveyed in maize, mouse and man. Nature 2003, 422(6929):297-302.

20. Schadt EE, Lamb J, Yang X, Zhu J, Edwards S, Guhathakurta D, Sieberts SK, Monks S, Reitman M, Zhang C, et al: An integrative genomics approach to infer causal associations between gene expression and disease. Nat Genet 2005, 37(7):710-717.

21. Yang X, Deignan JL, Qi H, Zhu J, Qian S, Zhong J, Torosyan G, Majid S, Falkard B, Kleinhanz RR, et al: Validation of candidate causal genes for obesity that affect shared metabolic pathways and networks. Nat Genet 2009, 41(4):415-423.

22. Ghazalpour A, Doss S, Zhang B, Wang S, Plaisier C, Castellanos R, Brozell A, Schadt EE, Drake TA, Lusis AJ, et al: Integrating genetic and network analysis to characterize genes related to mouse weight. PLoS Genet 2006, 2(8)-e130.

23. Plaisier CL, Horvath $\mathrm{S}$, Huertas-Vazquez A, Cruz-Bautista I, Herrera MF, TusieLuna T, Aguilar-Salinas C, Pajukanta P: A systems genetics approach implicates USF1, FADS3, and other causal candidate genes for familial combined hyperlipidemia. PLoS Genet 2009, 5(9)-e1000642.

24. Cahan P, Li Y, Izumi M, Graubert TA: The impact of copy number variation on local gene expression in mouse hematopoietic stem and progenitor cells. Nat Genet 2009, 41(4):430-437.

25. Bogue MA, Grubb SC, Maddatu TP, Bult CJ: Mouse Phenome Database (MPD). Nucleic Acids Res 2007, , 35 Database: D643-649.

26. Cahan P, Godfrey LE, Eis PS, Richmond TA, Selzer RR, Brent M, Mc Leod HL, Ley TJ, Graubert TA: wuHMM: a robust algorithm to detect DNA copy number variation using long oligonucleotide microarray data. Nucleic Acids Res 2008, 36(7)-e41.

27. Nadler JJ, Zou F, Huang H, Moy SS, Lauder J, Crawley JN, Threadgill DW, Wright FA, Magnuson TR: Large-scale gene expression differences across brain regions and inbred strains correlate with a behavioral phenotype. Genetics 2006, 174(3):1229-1236.

28. Breitling R, Li Y, Tesson BM, Fu J, Wu C, Wiltshire T, Gerrits A, Bystrykh LV, de Haan G, Su Al, et al: Genetical genomics: spotlight on QTL hotspots. PLoS Genet 2008, 4(10)-e1000232. 
29. Gerrits A, Li Y, Tesson BM, Bystrykh LV, Weersing E, Ausema A, Dontje B, Wang $X$, Breitling $R$, Jansen $R C$, et al: Expression quantitative trait loci are highly sensitive to cellular differentiation state. PLoS Genet 2009, 5(10)-e1000692

30. Zhang B, Horvath S: A general framework for weighted gene coexpression network analysis. Stat Appl Genet Mol Biol 2005, 4, Article17.

31. Langfelder $P$, Horvath S: WGCNA: an R package for weighted correlation network analysis. BMC Bioinformatics 2008, 9-559.

32. Stranger BE, Nica AC, Forrest MS, Dimas A, Bird CP, Beazley C, Ingle CE, Dunning $M$, Flicek $P$, Koller $D$, et al: Population genomics of human gene expression. Nat Genet 2007, 39(10):1217-1224.

33. Visel A, Zhu Y, May D, Afzal V, Gong E, Attanasio C, Blow MJ, Cohen JC, Rubin EM, Pennacchio LA: Targeted deletion of the 9p21 non-coding coronary artery disease risk interval in mice. Nature 464(7287):409-412

34. Mc Carroll SA, Kuruvilla FG, Korn JM, Cawley S, Nemesh J, Wysoker A, Shapero MH, de Bakker PI, Maller JB, Kirby A, et al: Integrated detection and population-genetic analysis of SNPs and copy number variation. Nat Genet 2008, 40(10):1166-1174

35. Young LC, Peters AC, Maeda T, Edelmann W, Kucherlapati R, Andrew SE, Tron VA: DNA mismatch repair protein Msh6 is required for optimal levels of ultraviolet-B-induced apoptosis in primary mouse fibroblasts. $J$ Invest Dermatol 2003, 121(4):876-880.

36. Roos WP, Christmann M, Fraser ST, Kaina B: Mouse embryonic stem cells are hypersensitive to apoptosis triggered by the DNA damage $\mathrm{O}(6)$ methylguanine due to high E2F1 regulated mismatch repair. Cell Death Differ 2007, 14(8):1422-1432.

37. Klapacz J, Meira LB, Luchetti DG, Calvo JA, Bronson RT, Edelmann W, Samson LD: O6-methylguanine-induced cell death involves exonuclease 1 as well as DNA mismatch recognition in vivo. Proc Natl Acad Sci USA 2009, 106(2):576-581

38. Hakem R, Hakem A, Duncan GS, Henderson JT, Woo M, Soengas MS, Elia A, de la Pompa JL, Kagi D, Khoo W, et al: Differential requirement for caspase 9 in apoptotic pathways in vivo. Cell 1998, 94(3):339-352.

39. Kuida K, Haydar TF, Kuan CY, Gu Y, Taya C, Karasuyama H, Su MS, Rakic P, Flavell RA: Reduced apoptosis and cytochrome c-mediated caspase activation in mice lacking caspase 9. Cell 1998, 94(3):325-337.

40. Marsden VS, O'Connor L, O'Reilly LA, Silke J, Metcalf D, Ekert PG, Huang DC, Cecconi F, Kuida K, Tomaselli KJ, et al: Apoptosis initiated by Bcl-2regulated caspase activation independently of the cytochrome c/Apaf-1/ caspase-9 apoptosome. Nature 2002, 419(6907):634-637.

41. Wu C, Orozco C, Boyer J, Leglise M, Goodale J, Batalov S, Hodge CL, Haase J, Janes J, Huss JW, et al: BioGPS: an extensible and customizable portal for querying and organizing gene annotation resources. Genome Biol 2009, 10(11):R130.

42. Seki A, Fang G: CKAP2 is a spindle-associated protein degraded by APC/ C-Cdh1 during mitotic exit. J Biol Chem 2007, 282(20):15103-15113.

43. Hong KU, Kim HJ, Kim HS, Seong YS, Hong KM, Bae CD, Park J: Cdk1-cyclin B1-mediated phosphorylation of tumor-associated microtubuleassociated protein/cytoskeleton-associated protein 2 in mitosis. $J$ Biol Chem 2009, 284(24):16501-16512.

44. Jeon SM, Choi B, Hong KU, Kim E, Seong YS, Bae CD, Park J: A cytoskeleton-associated protein, TMAP/CKAP2, is involved in the proliferation of human foreskin fibroblasts. Biochem Biophys Res Commun 2006, 348(1):222-228.

45. Schreiber V, Ame JC, Dolle P, Schultz I, Rinaldi B, Fraulob V, Menissier-de Murcia J, de Murcia G: Poly(ADP-ribose) polymerase-2 (PARP-2) is required for efficient base excision DNA repair in association with PARP1 and XRCC1. J Biol Chem 2002, 277(25):23028-23036.

46. Raffoul JJ, Cabelof DC, Nakamura J, Meira LB, Friedberg EC, Heydari AR: Apurinic/apyrimidinic endonuclease (APE/REF-1) haploinsufficient mice display tissue-specific differences in DNA polymerase beta-dependent base excision repair. J Biol Chem 2004, 279(18):18425-18433.

47. Yang $H$, Ding $Y$, Hutchins LN, Szatkiewicz J, Bell TA, Paigen BJ, Graber JH, de Villena FP, Churchill GA: A customized and versatile high-density genotyping array for the mouse. Nat Methods 2009, 6(9):663-666.

48. Sudbery I, Stalker J, Simpson JT, Keane T, Rust AG, Hurles ME, Walter K, Lynch D, Teboul L, Brown SD, et al: Deep short-read sequencing of chromosome 17 from the mouse strains A/J and CAST/Ei identifies significant germline variation and candidate genes that regulate liver triglyceride levels. Genome Biol 2009, 10(10):R112.
49. Quinlan AR, Clark RA, Sokolova S, Leibowitz ML, Zhang Y, Hurles ME, Mell JC, Hall IM: Genome-wide mapping and assembly of structural variant breakpoints in the mouse genome. Genome Res.

50. Rousseeuw PJ, Kaufman L: Finding Groups in Data: An Introduction to Cluster Analysis. New York: Wiley; 1990.

51. Szatkiewicz JP, Beane GL, Ding Y, Hutchins L, Pardo-Manuel de Villena F, Churchill GA: An imputed genotype resource for the laboratory mouse. Mamm Genome 2008, 19(3):199-208.

52. Zhao H, Nettleton D, Dekkers JC: Evaluation of linkage disequilibrium measures between multi-allelic markers as predictors of linkage disequilibrium between single nucleotide polymorphisms. Genet Res 2007, 89(1):1-6.

53. Smyth GK: Limma: linear models for microarray data. In Bioinformatics and Computational Biology Solutions using $R$ and Bioconductor. Edited by: $R$ Gentleman VC, Dudoit S, Irizarry R, Huber W. New York: Springer; 2005:397-420.

54. Smyth GK: Linear models and empirical bayes methods for assessing differential expression in microarray experiments. Stat Appl Genet Mol Biol 2004, 3, Article3.

55. Storey JD, Tibshirani R: Statistical significance for genomewide studies. Proc Natl Acad Sci USA 2003, 100(16):9440-9445.

56. Huang da W, Sherman BT, Lempicki RA: Systematic and integrative analysis of large gene lists using DAVID bioinformatics resources. Nat Protoc 2009, 4(1):44-57.

57. Mc Clurg P, Janes J, Wu C, Delano DL, Walker JR, Batalov S, Takahashi JS, Shimomura K, Kohsaka A, Bass J, et al: Genomewide association analysis in diverse inbred mice: power and population structure. Genetics 2007, 176(1):675-683.

58. Pletcher MT, Mc Clurg P, Batalov S, Su Al, Barnes SW, Lagler E, Korstanje R, Wang $X$, Nusskern D, Bogue MA, et al: Use of a dense single nucleotide polymorphism map for in silico mapping in the mouse. PLOS Biol 2004, 2(12):e393.

59. Mc Clurg P, Pletcher MT, Wiltshire T, Su Al: Comparative analysis of haplotype association mapping algorithms. BMC Bioinformatics 2006, 7:61.

60. Holm S: A simple sequentially rejective multiple test procedure. Scandinavian Journal of Statistics 1979, 6:65-70.

61. Benjamini $Y$, Hochberg $Y$ : Controlling the false discovery rate: A practical and powerful approach to multiple testing. J R Stat SOC B 1995, 57:289-300.

doi:10.1186/1471-2164-11-638

Cite this article as: Cahan and Graubert: Integrated genomics of susceptibility to alkylator-induced leukemia in mice. BMC Genomics 2010 $11: 638$.

\section{Submit your next manuscript to BioMed Central and take full advantage of:}

- Convenient online submission

- Thorough peer review

- No space constraints or color figure charges

- Immediate publication on acceptance

- Inclusion in PubMed, CAS, Scopus and Google Scholar

- Research which is freely available for redistribution

Submit your manuscript at www.biomedcentral.com/submit
C Biomed Central 